\title{
Rapid conversion of human ESCs into mouse ESC-like pluripotent state by optimizing culture conditions
}

\author{
Qi Gu ${ }^{1,2^{*}}$, Jie Hao ${ }^{1 *}$, Xiao-yang Zhao ${ }^{1}$, Wei Li ${ }^{1}$, Lei Liu ${ }^{1}$, Liu Wang ${ }^{1}$, Zhong-hua Liu ${ }^{2}$, Qi Zhou ${ }^{1 凶}$ \\ 1 State Key Laboratory of Reproductive Biology, Institute of Zoology, Chinese Academy of Sciences, Beijing 100101, China \\ ${ }^{2}$ College of Life Science, Northeast Agricultural University of China, Harbin 150030, China \\ \ Correspondence: qzhou@ioz.ac.cn
}

Received November 19, 2011 Accepted January 3, 2012

\begin{abstract}
The pluripotent state between human and mouse embryonic stem cells is different. Pluripotent state of human embryonic stem cells (ESCs) is believed to be primed and is similar with that of mouse epiblast stem cells (EpiSCs), which is different from the naïve state of mouse ESCs. Human ESCs could be converted into a naïve state through exogenous expression of defined transcription factors (Hanna et al., 2010). Here we report a rapid conversion of human ESCs to mouse ESC-like naïve states only by modifying the culture conditions. These converted human ESCs, which we called mhESCs (mouse ESC-like human ESCs), have normal karyotype, allow single cell passage, exhibit domed morphology like mouse ESCs and express some pluripotent markers similar with mouse ESCs. Thus the rapid conversion established a naïve pluripotency in human ESCs like mouse ESCs, and provided a new model to study the regulation of pluripotency.
\end{abstract}

KEYWORDS human embryonic stem cells (hESCs), mouse ESCs, naïve, pluripotent state

\section{INTRODUCTION}

Embryonic stem cells are the cells derived from the inner cell mass (ICM) of preimplantation blastocysts. Mouse and human embryonic stem cell lines were established in 1981 and 1998, respectively, for the first time (Evans and Kaufman, 1981; Martin, 1981; Thomson et al., 1998). In 2007, a new kind of pluripotent stem cell was established from postimplantation epibasts and named Epiblast stem cells

"These authors contributed equally to the work.
(EpiSCs). Mouse EpiSCs share many features with human ESCs but differ from mouse ESCs (Brons et al., 2007; Tesar et al., 2007). Thus mouse ESCs and EpiSCs are believed to have different pluripotent states: naïve and primed, respectively (Nichols and Smith, 2009). The naïve pluripotent stem cells contribute to the chimera at a high efficiency when injected into blastocysts, but the primed pluripotent stem cells cannot. Naïve pluripotent stem cells' self-renewal is dependent on leukemia inhibitory factor (LIF) and bone morphogenetic protein (BMP), but the primed pluripotent stem cells' self-renewal is dependent on basic fibroblast growth factor (bFGF) and Activin (Rossant, 2008). There are also many other different features between naïve and primed pluripotent stem cells. For example, one $\mathrm{X}$ chromosome in female mouse EpiSCs is inactivated while both $X$ chromosomes in female mouse ESCs are activated. Naïve pluripotent stem cells will be also differentiated under Fgf/Erk signals which can promote the self-renewal of primed pluripotent stem cells. In addition, they have some different markers and pluripotent factors (Nichols and Smith, 2009). LIF was thought to be essential for mouse ESCs self-renewal (Smith et al., 1988; Williams et al., 1988; Matsuda et al., 1999) and bFGF could inhibit some correlated factors to sustain undifferentiated state of human ESCs (Dvorak et al., 2005). BMP4 plays opposite roles in human ESCs and mouse ESCs: it could activate the gene ID which is essential for mouse ESCs but its expression promotes human ESCs differentiation into trophectoderm-like cells (Ying et al., 2003; Vallier et al., 2005). Besides relying on different pluripotent signaling pathways, the morphologies of human ESCs are also different from that of mouse ESCs. Mouse ESCs grow as three-dimensional domed colonies with well-rounded borders, and human ESCs colonies appear flatter, which are the same as mouse EpiSCs. Moreover, unlike mouse ESCs, passaging of mouse EpiSCs and human ESCs using single cell digestion 
would induce widespread cell death (Thomson et al., 1998; Tesar et al., 2007).

At present, mouse EpiSCs have been successfully converted into naïve pluripotent stem cells (Bao et al., 2009). EpiSCs have also been produced from ESCs by modifying culture agents (Guo et al., 2009). Moreover, mouse EpiSCs have been generated from preimplantation embryos (Najm et al., 2011). Human ESCs have reached a new pluripotent state with similar characters of mouse ESCs by exogenous expression of three pluripotent factors (Klf4, Oct4, Sox2), but the naïve pluripotent stem cell lines could not be maintained for longer than 20 passages without the expression of exogenes (Hanna et al., 2010). Small compounds (Erk inhibitor PD0325901, Gsk3 inhibitor CHIR99021, and adenyIylcyclase activator Forskolin) could convert human ESCs into naïve states, but domed colonies of naïve cells would form after 8-12 days of incubation in the medium containing small compounds and the naïve pluripotent stem cells could not be passaged stably (Hanna et al., 2010). Two compounds (Thiazovivin/Tzv and Tyrintegin/Ptn) were also reported to significantly increase single cell survival of human ESCs and promote domed colony formation; however, the authors did not further characterize the morphologically changed ESCs (Xu et al., 2010). In the current study, we report a rapid conversion of human ESCs into a naïve pluripotent state only by modifying the culture medium.

Culture media have profound effect on cell identity. Cell fate transition was shown to be induced or promoted by switching different culture media. A commercially available serum replacer (Knockout Serum Replacer, KSR) has been reported to promote the growth of pluripotent stem cells (Zhao et al., 2010). N2B27 medium was used to isolate rat embryonic stem cells (Buehr et al., 2008) and was successfully used in the establishment of rat naïve ESCs combined with Erk inhibitor PD0325901(PD) and Gsk3 inhibitor CHIR99021(CH) (Li et al., 2008). These two compounds both inhibit the pathways which promote differentiation and can help maintain self-renewal for the naïve pluripotent stem cells (Ying et al., 2008) and also have been indicated to promote mouse pluripotent state conversion (Guo et al., 2009; Hanna et al., 2009). LIF and bFGF respectively played important roles in maintaining mouse and human embryonic stem cell pluripotency (Williams et al., 1988; Bendall et al., 2007). The N2B27 medium containing bFGF was reported to be able to sustain human ESCs with the similar phenotype of that cultured in the traditional medium KOSR (Liu et al., 2006). Ascorbic acid (Buryanov and Shevchuk, 2005), a common nutrient for human health, could promote ESC proliferation (Chen et al., 2011) and was reported to promote reprogramming (Esteban et al., 2010). Human ESCs with two active $X$ chromosomes have been generated under physiological oxygen concentrations which could prevent cell senescence (Lengner et al., 2010). All of these agents may contribute to the conversion of the pluripotent cells from primed states to naïve states. In our study, we chose different combinations of basal media (KOSR, N2B27), factors (LIF, bFGF) and small compounds ( $\mathrm{PD}, \mathrm{CH}$, and $\mathrm{VC}$ ) to initially screen conditions that promote pluripotent state conversion. We revealed that the morphology and epigenetic markers of converted human ESCs (also called mhESCs) were closer to naïve states. Our findings provided satisfactory host cells for future transgenic medicine as well as a new method for human ESCs culture.

\section{RESULTS}

\section{Determining the optimized conversion condition}

The $\mathrm{H} 9$ cells were first digested into single cells (we named the passage for these cells $\mathrm{P} 0$ ) and planted on feeder cells using different media and at a density of $1 \times 10^{6}$ cells per dish. On the third day (D3), the domed colony emerged. A large number of colonies in the media containing N2B27 looked like mouse ESCs (Fig. 1A). But the cell colonies looked explanate and were only a little prominent in the KOSR medium with LIF, $\mathrm{LIF}+\mathrm{bFGF}$, or bFGF. On D3, all cells in different dishes were digested into single cells and seeded in the new medium respectively. After passaging (the passage was called $\mathrm{P} 1$ ), the single N2B27 medium could not maintain human ESCs pluripotency. Rather, they might have been differentiated. The media containing N2B27 and KOSR, LIF and bFGF or bFGF could continually sustain the growth of human ESCs with dome morphology. We compared the conversion efficiencies of different media by the colony number per unit area (CNpUA) (Fig. 1C). The media LBH (KOSR + LIF + bFGF), $\mathrm{BH}(\mathrm{KOSR}+\mathrm{bFGF}), \mathrm{LBX}(\mathrm{LIF}+\mathrm{bFGF}+\mathrm{KOSR}+\mathrm{N} 2 \mathrm{~B} 27)$ and $\mathrm{BX}(\mathrm{bFGF}+\mathrm{KOSR}+\mathrm{N} 2 \mathrm{~B} 27)$ converted $\mathrm{H} 9$ cells into mhESCs with a higher efficiency $(3.33 \pm 0.58,9.00 \pm 1.00$, $8.00 \pm 1.00$, respectively) (Fig. 1C) and the cells could proliferate with mouse ESCs morphology.

All the basal media contained the same small compounds ( $\mathrm{CH}, \mathrm{PD}, \mathrm{SB} 431542$, and VC). LBH, BH and LBX could support the converted $\mathrm{H} 9$ cells for more than 20 passages when digested with TrypLE ${ }^{\mathrm{TM}}$ (Invitrogen). The results also showed that all the media supporting the stable passage of mhESCs contained the factor bFGF. If the medium contained LIF, the colonies looked more domed than those in the media containing only bFGF (Fig. 1C). However, if the medium only contained the factor LIF, mhESCs would subsequently be differentiated in the following passages (Fig. 1B). The mhESCs in LBX medium had good phenotype (Fig. 1C), so we chose it to carry out the following experiments.

\section{Characterization of mESC-like human ESCs (mhESCs)}

There were many commonly used criteria to characterize and assess the developmental potential for the stem cells. Firstly, our karyotype analysis of mhESCs in LBX medium demonstrated that the converted human embryonic stem cells had a 


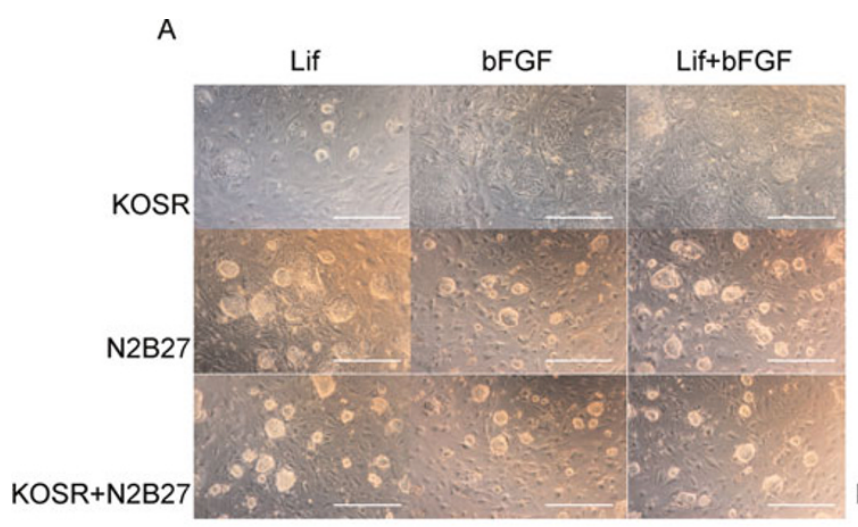

C

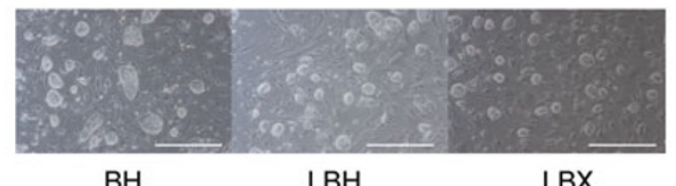

B
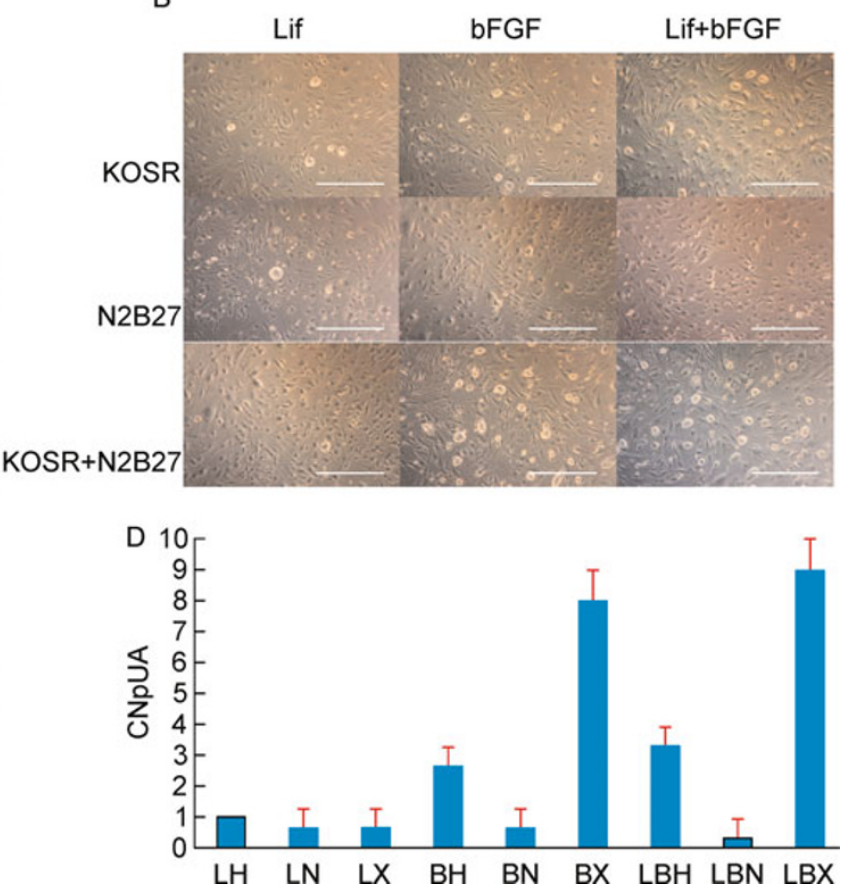

Figure 1. Determining the optimized conversion condition. (A) The PO mhESCs in different media. H9 cells (P37) with flat colonies were digested to single cells using Tryple ${ }^{T M}$ (Invitrogen). On the third day (3 days after incubation on feeders, named as D3), the colonies appeared as shown in the figure. (B) The P1 mhESCs on D2 in different media. The P0 mhESCs were digested on D3 of $\mathrm{P0}$. On the second day, the colonies of P1 mhESCs appeared as shown in the figure. (C) Cell morphology. The mhESCs at P20 in $\mathrm{BH}, \mathrm{LBH}$ and LBX media. The mhESCs in BH, LBH and LBX had been passaged for more than 20 generations. Bars $=500 \mu \mathrm{m}$. (D) Colony number per unit area (CNpUA) of mhESCs in different media, LN: LIF + N2B27; LH: LIF + KOSR; LBH: LIF + bFGF + KOSR; BN: bFGF + N2B27; LBN: LIF + bFGF + N2B27; LX: LIF + KOSR + N2B27; BX: bFGF + KOSR + N2B27; LBX: LIF + bFGF + KOSR + N2B27. The unit area was $1 \mathrm{~mm}^{2}$; the CNpUA of LN, LH, LBH, BN, LBN, LX, BX and LBX was $0.67 \pm 0.58,1.00 \pm 0.00,3.33 \pm 0.58$, $0.67 \pm 0.58,0.67 \pm 0.58,8.00 \pm 1.00,9.00 \pm 1.00$, respectively.

normal karyotype of $46(X X)$ (Fig. 2B). Next, we characterized the expression of some pluripotent markers in mhESCs. Specific markers for human ESCs (Tra-1-60, SSEA4) were still expressed (Fig. 2A). Moreover, the specific marker SSEA1 for mouse ESCs could also be stained in mhESCs (Fig. 2A). Later, we used RT-PCR and electrophoresis technology to detect some pluripotency genes expression, including Oct4, Sox2, Nanog, Lin28, Rex1, Gdf3 and Fgf4 and these genes all expressed in mhESCs as normally as the $\mathrm{H} 9$ cells (Fig. 2C). The assays of embryoid body (EB) formation in vitro were used to determine the differentiation ability of mhESCs. The mhESCs could form embryoid body-like structures through suspended growth (Fig. 2D) and RT-PCR analyses showed these bodies expressed differentiation markers for the three germ layers (Endoderm: Amylase and Ncstn, Mesoderm: Enolase and Osteonectin, Ectoderm: Gad1 and Gfap).

\section{The pluripotent state of mhESCs}

Real-time PCR analyses for the Xist gene (less expressed in naïve pluripotent stem cells (Nichols and Smith, 2009) were also performed to confirm that the converted mhESCs were similar to mouse ESCs. The expression of Xist (the first and second exons of Xist gene Xist12; the fifth and sixth exons of Xist gene Xist56 (Lengner et al., 2010)) was downregulated in the converted mhESCs cultured in LBH and LBX medium (Fig. 3A). SSEA4's expression was also found to be downregulated in the converted mhESCs (Fig. 3A). Furthermore, the staining of histone $\mathrm{H} 3$ lysine 27 trimethylation (H3K27me3), which is associated with the inactive $X$ chromosome (Plath et al., 2003), indicated that the accumulation of $\mathrm{H} 3 \mathrm{~K} 27 \mathrm{me} 3$ of $\mathrm{H} 9$ was more than that of mhESCs because there was no H3K27me3 'spot' in mhESCs (Fig. 3B). The copy of $X$ chromosomes which was inactivated in $\mathrm{H} 9$ cells might have been activated during the conversion. Thus, $\mathrm{H} 9$ cells might have been converted to naive pluripotent stem cells.

\section{Conversion of other human ESC lines}

We tried to convert human parthenogenetic embryonic stem 
A
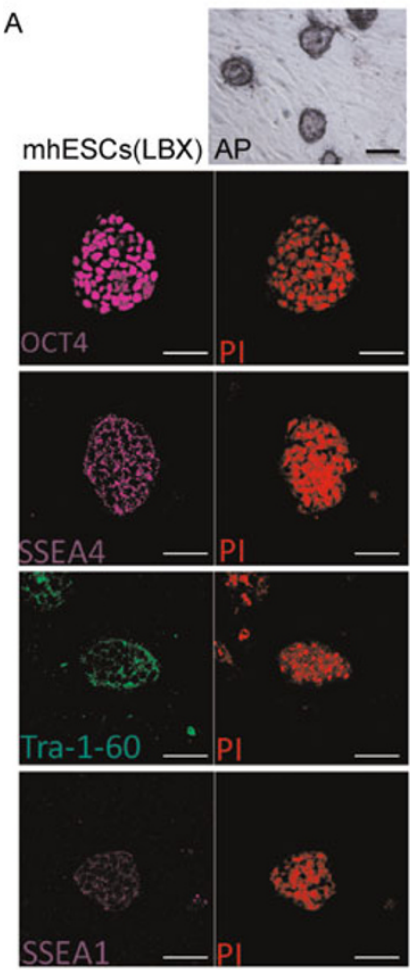

B

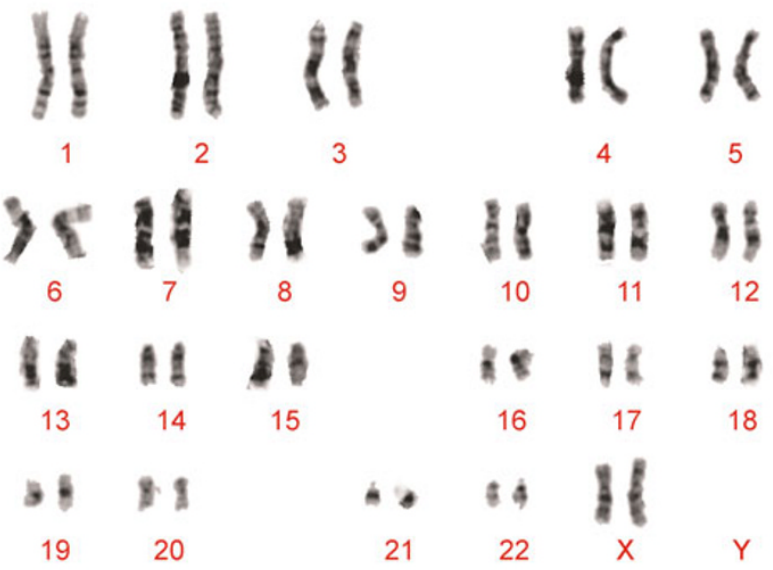

C

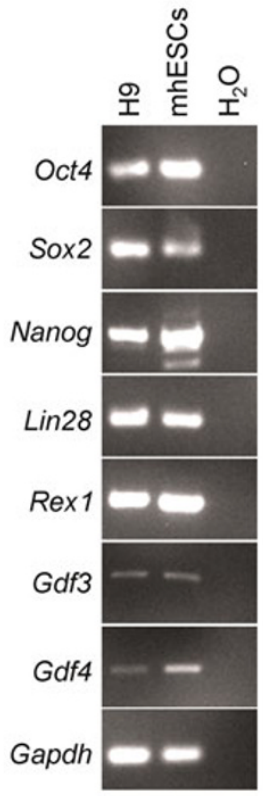

D

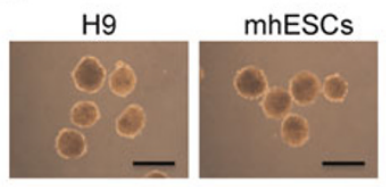

E

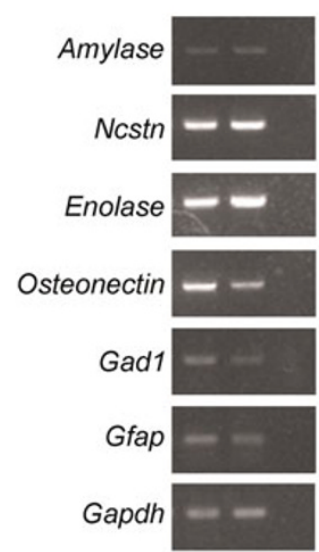

Figure 2. Characterization of mESC-like human ESCs (mhESCs). (A) Immunostaining for pluripotency markers (OCT4, SSEA1, SSEA4, and TRA-1-60) of mhESCs and H9 cells. AP, alkaline phosphatase. mhESCs in LBX medium and H9 cells in KOSR were stained with the same antibodies. Positive AP, Oct4, SSEA4 and Tra-1-60 were observed in H9 and mhESCs and SSEA1 is only positive in mhESCs. PI was used to stain the nucleus. Bars $=150 \mu \mathrm{m}$ (AP), Bars $=25 \mu \mathrm{m}$ (Immunostaining). (B) Karyotype of mhESCs. mhESCs in LBX medium were supplied for karyotype analysis. More than $75 \%$ of the cells showed normal human karyotype of 46 chromosomes. (C) RT-PCR for pluripotency genes (Oct4, Sox2, Nanog, Lin28, Gdf3 and Fgf4) of H9 and mhESCs. Seven pluripotency genes primers were supplied for PCR of $\mathrm{H} 9$, mhESCs and $\mathrm{H}_{2} \mathrm{O}$. (D) Embryoid body (EB) formation of $\mathrm{H} 9$ cells and mhESCs. Bars $=500 \mu \mathrm{m}$. (E) RT-PCR for three layers markers. The first column was the EB of H9 and the second and third columns were mhESCs' EBs and $\mathrm{H}_{2} \mathrm{O}$, respectively. 
A

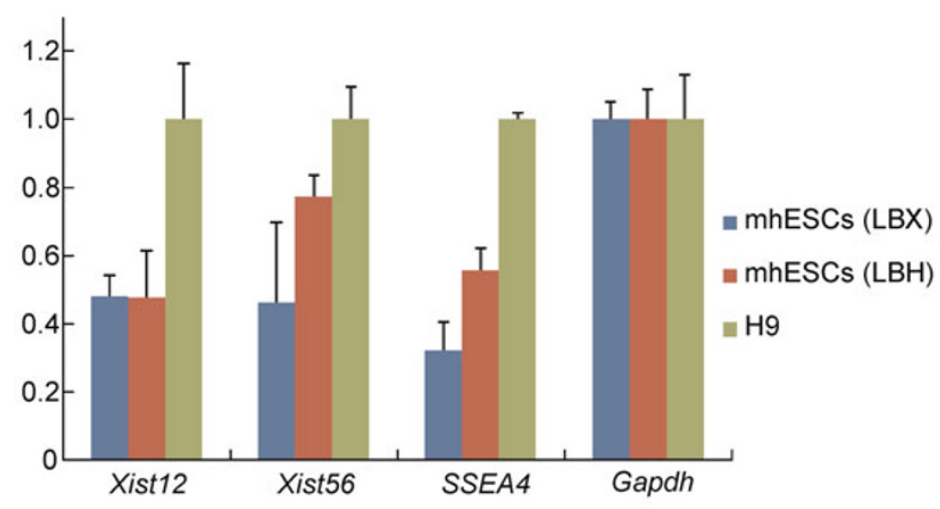

B

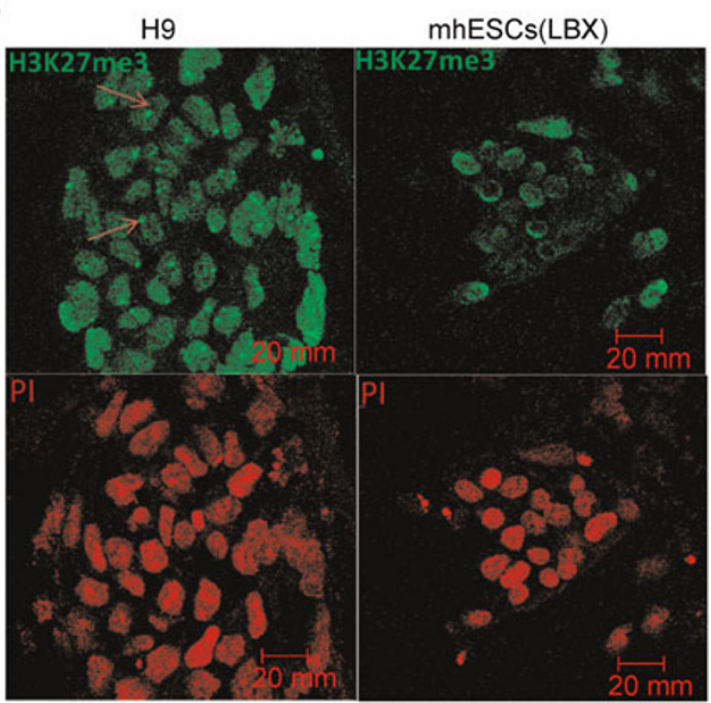

Figure 3. The pluripotent state of mhESCs. (A) Quantitative RT-PCR for Xist gene (Xist12 (Xist exons 1, 2), Xist56 (Xist exons 5 , 6)) and SSEA4. The expression of Xist and SSEA4 was relative to the expression of Gapdh. (B) H9 cells and mhESCs converted from $\mathrm{H} 9$ cells were stained with polyclonal antibodies against H3K27me3. Red arrow points to the H3K27me3 'spot'. PI was used to stain the nucleus. Bars $=20 \mu \mathrm{m}$.

cells (P-TJ cells (Lu et al., 2010)) into mouse ESC-like cells which we called m_P-TJ cells. The P-TJ cells displayed similar morphology as compared with $\mathrm{H} 9$ cells (Fig. 4A). P-TJ cells were also digested to single cells using Tryple $^{\mathrm{TM}}$ and
A

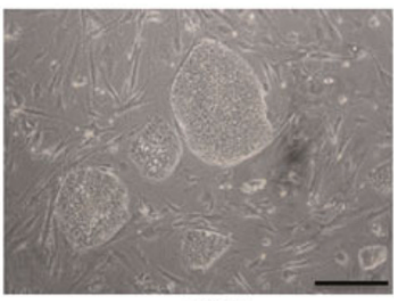

P-TJ

C

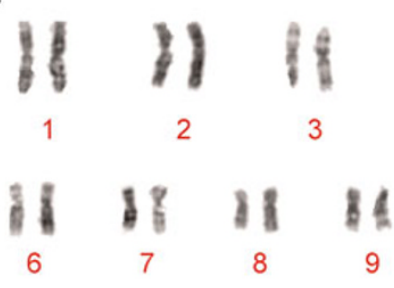

B

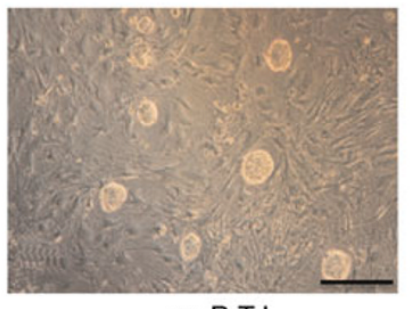

m_P-TJ

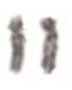

4
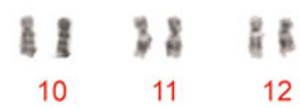

12

$\begin{array}{lll}k \text { z } & \text { a } \\ 16 & 17 & 18\end{array}$

$13 \quad 14 \quad 15$

88

19

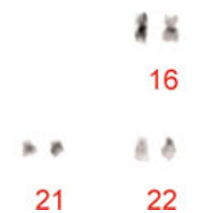

D

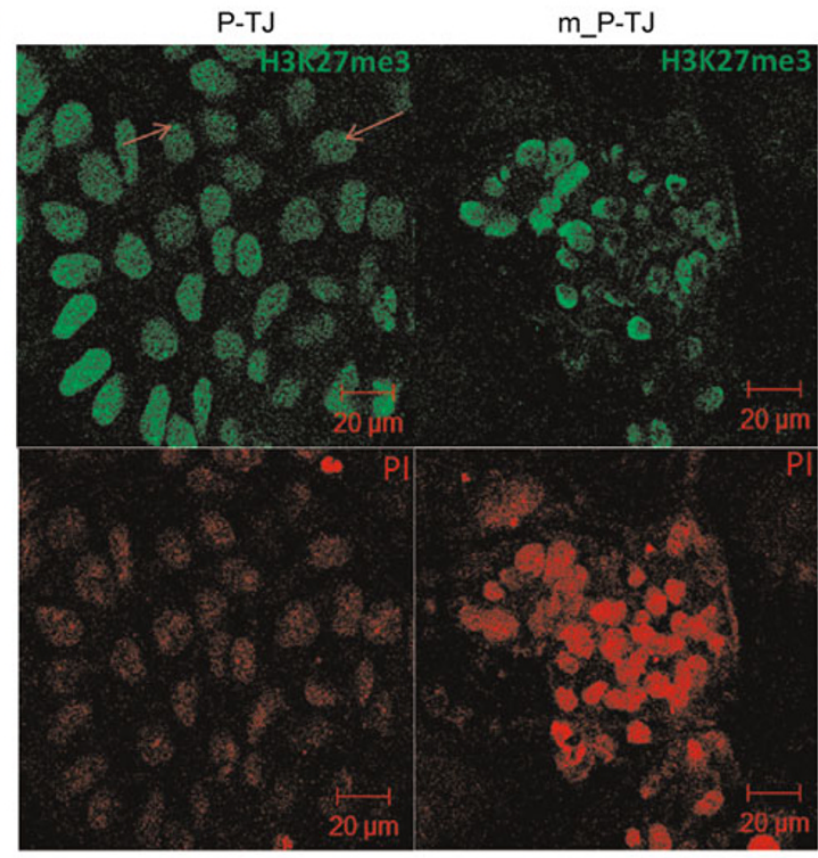

Figure 4. Conversion of other human ESC lines. (A) Cell morphology. P-TJ cells that were digested by collagenase IV. Bars = $500 \mu \mathrm{m}$. (B) Cell morphology. The mhESCs converted from P-TJ cells. Bars $=500 \mu \mathrm{m}$. (C) Karyotype analysis of m_P-TJ cells. More than $75 \%$ of the cells showed normal human karyotype of 46 chromosomes. (D) P-TJ cells and m_P-TJ cells were stained with polyclonal antibodies against $\mathrm{H} 3 \mathrm{~K} 27 \mathrm{me}$. Red arrow points to the $\mathrm{H} 3 \mathrm{~K} 27 \mathrm{me} 3$ 'spot'. PI was used to stain the nucleus. Bars $=20 \mu \mathrm{m}$. 
grew on feeders in LBX medium. On the third day after being digested, the colony grew bigger and could be digested for the second time and the converted cells had been digested for fifteen passages (Fig. 4B). The m_P-TJ cells had normal karyotype (Fig. 4C) and the H3K27me3 'spot' could be detected in P-TJ cells but its fluorescence intensity was weaker in m_P-TJ cells (Fig. 4D). So P-TJ cells might also have been converted to naïve pluripotent stem cells.

\section{DISCUSSION}

The H9 cells were quickly converted to naïve states with an alternative culture medium. Basal media played important roles in the fast conversion process because on $\mathrm{D} 3$ of $\mathrm{P} 0$, it was observed that colonies in the media containing N2B27 looked more domed than colonies in the media only containing KOSR, and the number of the colonies in the media containing N2B27 was also larger than that in the media only containing KOSR. Small compounds were also important to complete the conversion. At the early passages, the small compounds chosen were $\mathrm{CH}, \mathrm{PD}$ and VC. They could only support mhESCs for less than 3 passages and then the cells would prefer to differentiate and some went to apoptosis. The compound VC may accelerate the conversion process because it can promote cell proliferation (Chen et al., 2011). Another compound (activin receptor inhibitor SB431542, SB) was found by screening other small compounds (data not shown) and finally the small compounds array (CH, PD, SB, and VC) was determined. Activin receptor inhibitor SB was reported to increase BMP signaling activity (Xu et al., 2008) and induce rapid differentiation of EpiSCs (Brons et al., 2007). Therefore, all small compounds (CH, PD, SB, and VC) could support mhESCs in naive states by promoting self-renewal and proliferation of mhESCs without differentiation.

We converted human ESCs into mhESCs at a high efficiency and the converted cells could be passaged stably (Fig. 1). The mhESCs karyotype did not change during conversion (Fig. 2B and 4). The mhESCs still expressed some human ESCs specific markers but they could also express mouse ESCs specific markers (Fig. 2A). The expression of $X i s t$ gene can induce $\mathrm{X}$ chromosome inactivation $(\mathrm{XCl})$ and the region of $\mathrm{XCl}$ is accumulated by $\mathrm{H} 3-\mathrm{K} 27$ methylation (Plath et al., 2003). In our research, the expression of the Xist gene in mhESCs was downregulated and the fluorescence intensity of $\mathrm{H} 3 \mathrm{~K} 27 \mathrm{me} 3$ 'spot' in mhESCs was weaker than that in $\mathrm{H} 9$ and P-TJ cells (Fig. $3 B$ and $4 D$ ). So the mhESCs may have two active $X$ chromosomes $(\mathrm{XaXa})$ and the pluripotent state of mhESCs may be naïve.

Human ESC-based gene modifications would benefit much from the mhESCs. Homologous recombination works poor in human ESCs (Eiges et al., 2001) because of low colony formation efficiency of human ESCs which makes it difficult to screen complete positive colonies (Zwaka and
Thomson, 2003). When mhESCs were digested to single cells, the colonies would be formed from single cells, and the screening could also be performed by selecting the positive colonies generated from single cells. The positive cells could also be quickly screened by flow cytometry, which was useful for gene targeting and some other gene modification experiments. Furthermore, genetically modified human ESCs have particular application in establishing diseasespecific models (Li and Zhou, 2010) and the mhESCs will be the desirable materials.

Although the induced pluripotent stem cells of some species (mouse, human, monkey, rat, pig, rabbit, sheep, cow and dog) (Takahashi and Yamanaka, 2006; Takahashi et al., 2007; Liu et al., 2008; Ezashi et al., 2009; Liao et al., 2009; Honda et al., 2010; Bao et al., 2011; Han et al., 2011; Luo et al., 2011) and embryonic stem cells of some species (mouse, human, rat, monkey and rabbit) (Evans and Kaufman, 1981; Thomson et al., 1995; Thomson et al., 1998; Wang et al., 2007; Buehr et al., 2008) were established, most of these pluripotent stem cells were prone to be a primed pluripotent state rather than a naive state. The aim of the present experiment was to explore a medium suitable for obtaining naïve pluripotent stem cells. Our subsequent study is to obtain a general medium for as more species as possible. Further identification of the core transcriptional regulatory circuitry in mhESCs will provide us a comprehensive understanding of pluripotent state of human ESCs.

\section{MATERIALS AND METHODS}

\section{Reagents and media}

All the reagents and media used for this study were purchased from Life Technologies Inc. unless otherwise mentioned. H9 cells were from the Capital Medical University (CMU), Beijing; P-TJ cells were established in our labs (Lu et al., 2010). ICR mice for mouse embryonic stem cells (MEF) were purchased from Beijing Vital River Company. All animal operations followed the Guidelines for the Care and Use of Laboratory Animals established by the Beijing Association for Laboratory Animal Science, Beijing.

\section{Cell culture}

The KOSR medium consisted of $80 \%$ knockout Dulbecco's modified eagle medium (DMEM), 20\% knockout serum replacement, $2 \mathrm{mmol} / \mathrm{L}$ L-glutamine, $0.1 \mathrm{mmol} / \mathrm{L} \quad \beta$-mercaptoethanol (Sigma), 1\% MEM nonessential amino acids, $1 \%$ penicillin-streptomycin. N2B27 medium consisted of $48 \%$ DMEM/F12, 48\% Neurobasal, 1\% N2, 2\% B27, $0.25 \mathrm{mg} / \mathrm{mL}$ BSA (Sigma), $5 \mu \mathrm{g} / \mathrm{mL}$ insulin (Folch et al., 2009) and $1 \%$ penicillin-streptomycin.

Human ESCs were cultured as previously described (Hao et al., 2009). Feeder layer that was from the mouse embryonic fibroblast cells (MEF) treated with $10 \mu \mathrm{g} / \mathrm{mL}$ mitomycin and MEF were both cultured in $10 \%$ FBS DMEM medium and the human ESCs culture medium was KOSR of $8 \mathrm{ng} / \mathrm{mL}$ basic fibroblast growth factor (bFGF, $R \& D)$. After the clumps were dissociated mechanically for ten 
passages, they were passaged routinely using $1 \mathrm{mg} / \mathrm{mL}$ type IV collagenase (Sigma) every 6 days. All of the stem cells were cultured at $37^{\circ} \mathrm{C}$ in a $5 \% \mathrm{CO}_{2}$ incubator. The mhESCs were cultured in a different medium containing $2000 \mathrm{U} / \mathrm{mL}$ leucocyte inhibitory factor (LIF, Millipore) and $16 \mathrm{ng} / \mathrm{mL}$ basic fibroblast growth factor (bFGF, $R \& D)$. The mhESCs were passaged routinely using TrypLE ${ }^{\mathrm{TM}}$ (TrypLE ${ }^{\mathrm{TM}}$ Express \& TrypLE ${ }^{\mathrm{TM}}$ Select, Invitrogen) every 3 days. All the mhESCs media contained small compounds (Erk inhibitor

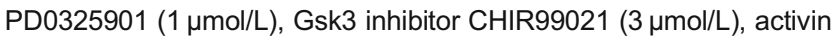
receptor inhibitor SB431542 $(2 \mu \mathrm{mol} / \mathrm{L})$ and ascorbic acid $(50 \mathrm{ng} / \mathrm{mL}))$.

\section{Colony number per unit area (CNpUA)}

mhESCs were planted on feeders at the same density when passaged from $\mathrm{P} 0$. On the second day, the colony number of the same area $\left(1 \mathrm{~mm}^{2}\right)$ for different media was counted and at least three different regions of the same area were selected. Variance analysis was performed. The mean value of the colony number for different regions in the same medium was named medium CNpUA.

\section{Immunofluorescence analysis and alkaline phosphatase}

Stained cells were fixed with $4 \%$ paraformaldehyde for $10 \mathrm{~min}$ and then permeabilized with $0.5 \%$ Triton $\mathrm{X}-100$ for $10 \mathrm{~min}$ followed by blocking with $5 \%$ donkey serum (Jakson) in PBS. The cells were incubated with primary antibodies against SSEA1 (Chemicon), OCT4 (Santa Cruz), TRA-1-60 (Chemicon), SSEA-4 (Chemicon) and $\mathrm{H} 3 \mathrm{~K} 27$ me3 (Chemicon) overnight at $4^{\circ} \mathrm{C}$, followed by the use of secondary antibodies at room temperature for $1 \mathrm{~h}$. Finally, DNA was stained with propidium iodide $(10 \mu \mathrm{g} / \mathrm{mL}$; Molecular Probes, OR) for $5 \mathrm{~min}$. For each separate sample, the experimental procedure was repeated for three times for one antibody, and the experiments were replicated for at least three times. Observations were made using a confocal microscope (Zeiss, LSM 510 META). Alkaline phosphatase (AP) staining was performed with BCIP/NBT Alkaline Phosphatase Colour Development Kit (Beyotime) following the manufacturer's instructions.

\section{Karyotype analysis}

Karyotype analysis was performed as previously described following the standard instructions (Zhao et al., 2009); G-binding was carried out by Peking Union Medical College, Beijing.

\section{Embryoid body formation}

For EB formation, the mhESCs were digested into single cells and were transferred to gelatin-coated plates and incubated in DMEM containing $10 \%$ FBS for $10 \mathrm{~min}$ to discard the feeder cells. Then the suspensions were harvested and transferred to poly(2-hydroxyrthyl methacrylate)-coated dish in KOSR medium lacking bFGF. The medium was changed every other day and after 6 days, the EBs were collected.

\section{RT-PCR and quantitative PCR analysis}

Total RNA was isolated from freshly obtained cells using TRIzol reagent. First strand cDNA was synthesized using MMLV reverse transcriptase (Promega) and oligo-dT (Promega) according to the manufacturer's instructions. RT-PCR was performed according to standard procedures and the products were electrophoresed on a $2 \%$ agarose gel. QPCR was performed using SYBR Green Real-time PCR Master Mix (Toyobo)on Agilent Mx3005P. All primers used in the research are listed in Table 1.

Table 1 Primer sequence for PCR

\begin{tabular}{lll}
\hline Gene name & \multicolumn{1}{c}{ Forward primers } & \multicolumn{1}{c}{ Reverse primers } \\
\hline Xist12 & GAAGAGTCTCTGGCTCTTTAGAATACTGA & CAGCGTGGTATCTTCAATGGG (Lengner et al., 2010) \\
SsEA4 & GCCTGGCACTCTAGCACTTGA & AGGAGACAAAGAATACACATTCATTC (Lengner et al., 2010) \\
Oct4 & GACAGGGGCACAACTTCATC & GGGCAGGTTCTTGGCACTCT \\
Sox2 & GGGAAATGGGAGGGGTGCAAAAGAGG & CTTCCCTCCAACCAGTTGCCCCAAAC \\
Nanog & CAGCCCCGATTCTTCCACCAGTCCC & CGGAAGATTCCCAGTCGGGTTCACC \\
Lin28 & GCAGAAGATCACTCCGTTCCA & CGCACATTGAACCACTTACAGT \\
Rex1 & CAGATCCTAAACAGCTCGCAGAAT & GCGTACGCAAATTAAAGTCCAGA \\
Gdf3 & CTTATGCTACGTAAAGGAGCTGGG & GTGCCAACCCAGGTCCCGGAAGTT \\
Fgf4 & CTACAACGCCTACGAGTCCTACA & GTTGCACCAGAAAATCAGAGTTG \\
Amylase & AATGATGCTACTCAGGTCAGAGATTGTC & TGTCCTCGTTGATTGTCATGGTTATCC \\
Ncstn & CGAGGATGGTCTACGATATGGAGAAGG & TCAGCCAGAACAACGCCAGAGAT \\
Enolase & GCTCCGTGACCGAGTCTCTT & TAGCCAACAGGTGACCGAAGG \\
Osteonectin & CCAGGTGGAAGAGGAGATT & CTCAGTCAGAAGGTTGTTGTC \\
Gad1 & GGAACTAGCGAGAACGAGGAAG & AGGAGGTTGCGGACGAAGAT \\
Gfap & TGAGTCGCTGGAGGAGGGAT & GTCGTTGGCTTCGTGCTTGG \\
Gapdh & CGCTTCGCTCTCTGCTCCTCCTGTT & CGCTTCGCTCTCTGCTCCTCCTGTT \\
\hline
\end{tabular}




\section{ACKNOWLEDGEMENTS}

This work was supported in part by grants from the China National Basic Research Program (Grant No. 2011CB965300) to L.W. and grants from the National Natural Science Foundation of China (Grant No. 90919060) to Q.Z. and grants from the "Strategic Priority Research Program" of the Chinese Academy of Sciences (No. XDA01020101) to Q.Z.

\section{ABBREVIATIONS}

bFGF, basic fibroblast growth factor; BMP, bone morphogenetic protein; EpiSCs, mouse epiblast stem cells; ESCs, embryonic stem cells; mhESCs, mouse ESC-like human ESCs; ICM, inner cell mass; LIF, leukemia inhibitory factor; MEF, mouse embryonic fibroblast cells; $\mathrm{XCl}, \mathrm{X}$ chromosome inactivation

\section{REFERENCES}

Bao, L., He, L., Chen, J., Wu, Z., Liao, J., Rao, L., Ren, J., Li, H., Zhu, H., Qian, L., et al. (2011). Reprogramming of ovine adult fibroblasts to pluripotency via drug-inducible expression of defined factors. Cell Res 21, 600-608.

Bao, S., Tang, F., Li, X., Hayashi, K., Gillich, A., Lao, K., and Surani, M.A. (2009). Epigenetic reversion of post-implantation epiblast to pluripotent embryonic stem cells. Nature 461, 1292-1295.

Bendall, S.C., Stewart, M.H., Menendez, P., George, D., Vijayaragavan, K., Werbowetski-Ogilvie, T., Ramos-Mejia, V., Rouleau, A., Yang, J., Bossé, M., et al. (2007). IGF and FGF cooperatively establish the regulatory stem cell niche of pluripotent human cells in vitro. Nature 448, 1015-1021.

Brons, I.G., Smithers, L.E., Trotter, M.W., Rugg-Gunn, P., Sun, B., Chuva de Sousa Lopes, S.M., Howlett, S.K., Clarkson, A., AhrlundRichter, L., Pedersen, R.A., et al. (2007). Derivation of pluripotent epiblast stem cells from mammalian embryos. Nature 448 , 191-195.

Buehr, M., Meek, S., Blair, K., Yang, J., Ure, J., Silva, J., McLay, R., Hall, J., Ying, Q.L., and Smith, A. (2008). Capture of authentic embryonic stem cells from rat blastocysts. Cell 135, 1287-1298.

Buryanov, Y.I., and Shevchuk, T.V. (2005). DNA methyltransferases and structural-functional specificity of eukaryotic DNA modification. Biochemistry (Mosc) 70, 730-742.

Chen, G., Gulbranson, D.R., Hou, Z., Bolin, J.M., Ruotti, V., Probasco, M.D., Smuga-Otto, K., Howden, S.E., Diol, N.R., Propson, N.E., et al. (2011). Chemically defined conditions for human iPSC derivation and culture. Nat Methods 8, 424-429.

Dvorak, P., Dvorakova, D., Koskova, S., Vodinska, M., Najvirtova, M., Krekac, D., and Hampl, A. (2005). Expression and potential role of fibroblast growth factor 2 and its receptors in human embryonic stem cells. Stem Cells 23, 1200-1211.

Eiges, R., Schuldiner, M., Drukker, M., Yanuka, O., Itskovitz-Eldor, J., and Benvenisty, N. (2001). Establishment of human embryonic stem cell-transfected clones carrying a marker for undifferentiated cells. Curr Biol 11, 514-518.

Esteban, M.A., Wang, T., Qin, B., Yang, J., Qin, D., Cai, J., Li, W., Weng, Z., Chen, J., Ni, S., et al. (2010). Vitamin C enhances the generation of mouse and human induced pluripotent stem cells. Cell Stem Cell 6, 71-79.
Evans, M.J., and Kaufman, M.H. (1981). Establishment in culture of pluripotential cells from mouse embryos. Nature 292, 154-156.

Ezashi, T., Telugu, B.P., Alexenko, A.P., Sachdev, S., Sinha, S., and Roberts, R.M. (2009). Derivation of induced pluripotent stem cells from pig somatic cells. Proc Natl Acad Sci U S A 106, 10993-10998.

Folch, J., Cocero, M.J., Chesné, P., Alabart, J.L., Domínguez, V., Cognié, Y., Roche, A., Ferníndez-Arias, A., Martí, J.I., Sánchez, P., et al. (2009). First birth of an animal from an extinct subspecies (Capra pyrenaica pyrenaica) by cloning. Theriogenology 71 , 1026-1034.

Guo, G., Yang, J., Nichols, J., Hall, J.S., Eyres, I., Mansfield, W., and Smith, A. (2009). Klf4 reverts developmentally programmed restriction of ground state pluripotency. Development 136, 1063-1069.

Han, X., Han, J., Ding, F., Cao, S., Lim, S.S., Dai, Y., Zhang, R., Zhang, Y., Lim, B., and Li, N. (2011). Generation of induced pluripotent stem cells from bovine embryonic fibroblast cells. Cell Res 21, 1509-1512.

Hanna, J., Cheng, A.W., Saha, K., Kim, J., Lengner, C.J., Soldner, F., Cassady, J.P., Muffat, J., Carey, B.W., and Jaenisch, R. (2010). Human embryonic stem cells with biological and epigenetic characteristics similar to those of mouse ESCs. Proc Natl Acad Sci U S A.

Hanna, J., Markoulaki, S., Mitalipova, M., Cheng, A.W., Cassady, J. P., Staerk, J., Carey, B.W., Lengner, C.J., Foreman, R., Love, J., et al. (2009). Metastable pluripotent states in NOD-mouse-derived ESCs. Cell Stem Cell 4, 513-524.

Hao, J., Zhu, W., Sheng, C., Yu, Y., and Zhou, Q. (2009). Human parthenogenetic embryonic stem cells: one potential resource for cell therapy. Sci China C Life Sci 52, 599-602.

Honda, A., Hirose, M., Hatori, M., Matoba, S., Miyoshi, H., Inoue, K., and Ogura, A. (2010). Generation of induced pluripotent stem cells in rabbits: potential experimental models for human regenerative medicine. J Biol Chem 285, 31362-31369.

Lengner, C.J., Gimelbrant, A.A., Erwin, J.A., Cheng, A.W., Guenther, M.G., Welstead, G.G., Alagappan, R., Frampton, G.M., Xu, P., Muffat, J., et al. (2010). Derivation of pre-X inactivation human embryonic stem cells under physiological oxygen concentrations. Cell 141, 872-883.

Li, P., Tong, C., Mehrian-Shai, R., Jia, L., Wu, N., Yan, Y., Maxson, R. E., Schulze, E.N., Song, H., Hsieh, C.L., et al. (2008). Germline competent embryonic stem cells derived from rat blastocysts. Cell 135, 1299-1310.

Li, Z.K., and Zhou, Q. (2010). Cellular models for disease exploring and drug screening. Protein Cell 1, 355-362.

Liao, J., Cui, C., Chen, S., Ren, J., Chen, J., Gao, Y., Li, H., Jia, N., Cheng, L., Xiao, H., et al. (2009). Generation of induced pluripotent stem cell lines from adult rat cells. Cell Stem Cell 4, 11-15.

Liu, H., Zhu, F., Yong, J., Zhang, P., Hou, P., Li, H., Jiang, W., Cai, J., Liu, M., Cui, K., et al. (2008). Generation of induced pluripotent stem cells from adult rhesus monkey fibroblasts. Cell Stem Cell 3, 587-590.

Liu, Y., Song, Z., Zhao, Y., Qin, H., Cai, J., Zhang, H., Yu, T., Jiang, S., Wang, G., Ding, M., et al. (2006). A novel chemical-defined medium with bFGF and N2B27 supplements supports undifferentiated growth in human embryonic stem cells. Biochem Biophys Res Commun 346, 131-139. 
Lu, Z., Zhu, W., Yu, Y., Jin, D., Guan, Y., Yao, R., Zhang, Y.A., Zhang, Y., and Zhou, Q. (2010). Derivation and long-term culture of human parthenogenetic embryonic stem cells using human foreskin feeders. J Assist Reprod Genet 27, 285-291.

Luo, J., Suhr, S.T., Chang, E.A., Wang, K., Ross, P.J., Nelson, L.L., Venta, P.J., Knott, J.G., and Cibelli, J.B. (2011). Generation of leukemia inhibitory factor and basic fibroblast growth factordependent induced pluripotent stem cells from canine adult somatic cells. Stem Cells Dev 20, 1669-1678.

Martin, G.R. (1981). Isolation of a pluripotent cell line from early mouse embryos cultured in medium conditioned by teratocarcinoma stem cells. Proc Natl Acad Sci U S A 78, 7634-7638.

Matsuda, T., Nakamura, T., Nakao, K., Arai, T., Katsuki, M., Heike, T., and Yokota, T. (1999). STAT3 activation is sufficient to maintain an undifferentiated state of mouse embryonic stem cells. EMBO J 18, 4261-4269.

Najm, F.J., Chenoweth, J.G., Anderson, P.D., Nadeau, J.H., Redline, R.W., McKay, R.D., and Tesar, P.J. (2011). Isolation of epiblast stem cells from preimplantation mouse embryos. Cell Stem Cell 8 , 318-325.

Nichols, J., and Smith, A. (2009). Naive and primed pluripotent states. Cell Stem Cell 4, 487-492.

Plath, K., Fang, J., Mlynarczyk-Evans, S.K., Cao, R., Worringer, K.A., Wang, H., de la Cruz, C.C., Otte, A.P., Panning, B., and Zhang, Y. (2003). Role of histone $\mathrm{H} 3$ lysine 27 methylation in $X$ inactivation. Science 300, 131-135.

Rossant, J. (2008). Stem cells and early lineage development. Cell 132, 527-531.

Smith, A.G., Heath, J.K., Donaldson, D.D., Wong, G.G., Moreau, J., Stahl, M., and Rogers, D. (1988). Inhibition of pluripotential embryonic stem cell differentiation by purified polypeptides. Nature 336, 688-690.

Takahashi, K., Tanabe, K., Ohnuki, M., Narita, M., Ichisaka, T., Tomoda, K., and Yamanaka, S. (2007). Induction of pluripotent stem cells from adult human fibroblasts by defined factors. Cell 131, 861-872.

Takahashi, K., and Yamanaka, S. (2006). Induction of pluripotent stem cells from mouse embryonic and adult fibroblast cultures by defined factors. Cell 126, 663-676.

Tesar, P.J., Chenoweth, J.G., Brook, F.A., Davies, T.J., Evans, E.P., Mack, D.L., Gardner, R.L., and McKay, R.D. (2007). New cell lines from mouse epiblast share defining features with human embryo- nic stem cells. Nature 448, 196-199.

Thomson, J.A., Itskovitz-Eldor, J., Shapiro, S.S., Waknitz, M.A., Swiergiel, J.J., Marshall, V.S., and Jones, J.M. (1998). Embryonic stem cell lines derived from human blastocysts. Science 282 , 1145-1147.

Thomson, J.A., Kalishman, J., Golos, T.G., Durning, M., Harris, C.P., Becker, R.A., and Hearn, J.P. (1995). Isolation of a primate embryonic stem cell line. Proc Natl Acad Sci U S A 92, 7844-7848.

Vallier, L., Alexander, M., and Pedersen, R.A. (2005). Activin/Nodal and FGF pathways cooperate to maintain pluripotency of human embryonic stem cells. J Cell Sci 118, 4495-4509.

Wang, S., Tang, X., Niu, Y., Chen, H., Li, B., Li, T., Zhang, X., Hu, Z., Zhou, Q., and Ji, W. (2007). Generation and characterization of rabbit embryonic stem cells. Stem Cells 25, 481-489.

Williams, R.L., Hilton, D.J., Pease, S., Willson, T.A., Stewart, C.L., Gearing, D.P., Wagner, E.F., Metcalf, D., Nicola, N.A., and Gough, N.M. (1988). Myeloid leukaemia inhibitory factor maintains the developmental potential of embryonic stem cells. Nature 336 , 684-687.

Xu, R.H., Sampsell-Barron, T.L., Gu, F., Root, S., Peck, R.M., Pan, G., Yu, J., Antosiewicz-Bourget, J., Tian, S., Stewart, R., et al. (2008). NANOG is a direct target of TGFbeta/activin-mediated SMAD signaling in human ESCs. Cell Stem Cell 3, 196-206.

Xu, Y., Zhu, X., Hahm, H.S., Wei, W., Hao, E., Hayek, A., and Ding, S. (2010). Revealing a core signaling regulatory mechanism for pluripotent stem cell survival and self-renewal by small molecules. Proc Natl Acad Sci U S A 107, 8129-8134.

Ying, Q.L., Nichols, J., Chambers, I., and Smith, A. (2003). BMP induction of Id proteins suppresses differentiation and sustains embryonic stem cell self-renewal in collaboration with STAT3. Cell 115, 281-292.

Ying, Q.L., Wray, J., Nichols, J., Batlle-Morera, L., Doble, B., Woodgett, J., Cohen, P., and Smith, A. (2008). The ground state of embryonic stem cell self-renewal. Nature 453, 519-523.

Zhao, X.Y., Li, W., Lv, Z., Liu, L., Tong, M., Hai, T., Hao, J., Guo, C.L., Ma, Q.W., Wang, L., et al. (2009). iPS cells produce viable mice through tetraploid complementation. Nature 461, 86-90.

Zhao, X.Y., Lv, Z., Li, W., Zeng, F., and Zhou, Q. (2010). Production of mice using iPS cells and tetraploid complementation. Nat Protoc 5, 963-971.

Zwaka, T.P., and Thomson, J.A. (2003). Homologous recombination in human embryonic stem cells. Nat Biotechnol 21, 319-321. 